ORIGINAL ARTICLE

\title{
Anxiety and depressive disorders in an emergency department ward of a general hospital: a control study
}

\author{
C Marchesi, E Brusamonti, C Borghi, A Giannini, R Di Ruvo, F Minneo, C Quarantelli, C Maggini
}

Emerg Med J 2004;21:175-179. doi: 10.1136/emj.2003.006957

See end of article for authors' affiliations

.....................

Correspondence to:

Dr M Carlo, Dipartimento

di Neuroscienze, Sezione

di Psichiatrica, Universitò

di Parma, Strada del

Quartiere 2,43100

Parma, Italy;

carlo.marchesi@unipr.it

Accepted for publication

3 June 2003

\begin{abstract}
Objective: In this study anxiety and depressive disorders were evaluated in patients admitted to an emergency department (ED) or to a medical department (MD).

Methods: The General Health Questionnaire-30 (GHQ-30) was administered to screen all patients $(n=719)$ consecutively admitted to an $E D(n=556)$ and to $M D(n=163)$ in a 120 day period. All GHQ-30 positive (score $>4$ ) underwent the Mini International Neuropsychiatric Interview, a structured interview to diagnose mental disorders according to DSM-IV criteria.

Results: Subjects positive to GHQ-30 were $264(47 \%)$ in ED and 88 (54\%) in MD. A mental disorder was diagnosed in $233 \mathrm{ED}$ patients $(42 \%)$ and in $77 \mathrm{MD}$ patients $(47 \%)(p=0.70)$. The most frequent disorders were anxiety disorders in ED patients (18.1\%) and depressive disorders in MD patients $(21 \%)(p=0.04)$. Conclusions: Anxious patients more frequently seek attention at ED, whereas patients with depressive disorders are more often observed in medical units. The improvement of quality of care, the waste of healthcare resources through unnecessary medical care, and the well known efficacy of appropriate treatments in patients with anxiety and depressive disorders make the diagnosis of these patients particularly important.
\end{abstract}

n a general hospital, patients with psychiatric disorders are commonly found ${ }^{1-6}$ with a prevalence varying from $30 \%$ to $60 \%{ }^{1}{ }^{7-9}$ Previous studies seem to suggest that anxiety disorders are expected to be frequent in emergency departments (ED) and depressive disorders in medical departments (MD). However, these studies evaluated only the prevalence of panic disorder in $\mathrm{ED}^{10-17}$ and major depression in $\mathrm{MD},{ }_{1}{ }^{18-22}$ whereas none of them have compared the rate of anxiety and depressive disorders in ED and MD. Therefore, to verify whether anxiety and depressive disorders are differently distributed in ED and in MD, we compared the psychiatric morbidity in patients admitted to ED and to MD. Such information may help to recognise psychiatric disorders in a general hospital, to improve quality of care, and to reduce the cost of the treatment, avoiding unnecessary medical treatments.

\section{METHODS}

The study was approved by the ethics committee of the Parma University Hospital.

\section{Sample}

The patients included in the study were selected from all subjects who had been consecutively admitted to the ED and MD wards of the General Hospital of Parma, Italy, during a 120 days period (May to September 1996) if: (1) their age was between 18 and 65 years; (2) they had completed the diagnostic interview and the psychopathological evaluations; (3) their written informed consent had been obtained.

We excluded from the study: (1) subjects older than 65, because organic mental disorders, such as dementia and delirium are quite common in this population, but it is difficult to accurately measure their frequency. In fact, many patients with cognitive disorders are unable to give their informed consent or to complete questionnaires; (2) subjects who were too ill, either mentally of physically, to complete questionnaires; (3) subjects unable to speak Italian.

The General Hospital of Parma is a University Hospital with 1600 beds, serving a population of 400000 people.
In Italy, ED wards can be considered as an acute medical ward where patients were admitted and stay for short periods of time (see below) for a diagnostic check up and treatment. Therefore, in Italy the difference between patients visited at the ED (who go home in few hours after physician consultation) and patients admitted to the ED ward (who are hospitalised for few days) is attributable to their physical condition. Moreover, two different medical and nursing staffs are involved in ED outpatient service and in ED inpatient ward.

Emergency patients with overt psychiatric symptoms were referred by the emergency physicians to the psychiatric ED for a consultation. After consultation, patients were admitted to the psychiatric ward or were given a psychiatric outpatient appointment, according to the severity of their illness. On the other hand, patients with alcohol or drug misuse or dependence were either admitted to other units because of their physical or mental conditions or sent to the Unit for the Treatment of Alcohol and Drug Dependence. Patients who could not be admitted to the unit (because of patient refusal or bed not available) were admitted to the ED ward for an acute detoxification treatment.

During 1996, 41500 patients were visited at the ED, and 2745 patients were admitted to the ED ward for a mean period of 2.4 days (in the same year, the mean period of hospitalisation in medical wards was 10.3 days).

\section{Assessment: Stage 1}

GHQ- $30^{23}$ was administered to all subjects after collection of their basic sociodemographic data (age, sex, marital and family status, education, occupation).

GHQ-30 is a self administered questionnaire with 30 items evaluating general health, sleep and wakefulness, personal

\footnotetext{
Abbreviations: $E D$, emergency department; $M D$, medical department; GHQ-3, General Health Questionnaire-30; MINI, Mini International Neuropsychiatric Interview; DUSOI, Duke Severity of Illness
} 
and social behaviours, subjective feelings (inadequacy, tension, anxiety, depression). The answers to each item consist in the following four point scale: "not at all", "no more than usual", "rather more than usual", "much more than usual". The method of scoring the questionnaire is to assign a score of zero to responses 1 and 2, and a score of unity to responses 3 and 4 (modified Likert scoring). A total score higher than 4 identifies subjects presenting a psychological distress (referred in the text as GHQ-30 positive patients).

In our previous study, ${ }^{24}$ a GHQ-30 total score higher than 4 showed a sensitivity of $88.6 \%$, a specificity of $87.5 \%$, and a positive predictive value of $95.5 \%$ in detecting patients with DSM-IV ${ }^{25}$ mental disorders in a GH.

\section{Stage 2}

All GHQ-30 positive patients underwent a clinical psychiatric examination, after giving their informed consent. During the clinical examination, the Italian version ${ }^{26}$ of the Mini International Neuropsychiatric Interview, ${ }^{27}$ was administered. This structured interview provides the possibility to establish in a short time (about 20 minutes) the diagnosis of Major Depression, Dysthymia, Mania and Hypomania, Panic Disorder, Agoraphobia, Social Phobia, Obsessive-Compulsive Disorder, Generalised Anxiety Disorder, Substance Abuse and Dependence, Psychotic Disorders, Anorexia and Bulimia Nervosa, Somatisation Disorder, Adjustment Disorders according to DSM-IV criteria. ${ }^{25}$ Furthermore, the presence of Minor Depressive Disorder and Mixed Anxiety-Depressive Disorder was investigated using the research diagnostic criteria listed in the appendix B ("Criteria sets and axes provided for further study") of the DSM-IV ${ }^{25}$ (pages 720 and 724).

A psychiatrist (EB) was specifically trained to administer the MINI. The training consisted in the administration of the MINI to 10 patients affected by Major Depression, Dysthymia, Panic Disorder, Generalized Anxiety Disorder, Mixed Anxiety-Depressive Disorder. The interviews were audiotaped and reviewed by two psychiatrists involved in the preparation of the Italian version of the MINI. A satisfactory reliability $(\kappa=0.76)$ was obtained during the training. The psychiatric diagnoses of patients included in the study were also discussed with a senior psychiatrist (CM).

In all patients, the severity of the illnesses was evaluated by the physicians of the ED and MD wards using the Duke Severity of Illness (DUSOI) ${ }^{28}$ and the diagnoses at discharge were registered using the hospital data base.

\section{Statistical analysis}

Comparisons between group were made with the $\chi^{2}$ test for categorical variables and with one way analysis of variance and the two tailed Student's t test for continuous variables, as appropriate. Statistical analyses were performed with SPSS 10.0 release.

\section{RESULTS}

Sample

We excluded 167 subjects from the study: 103 patients were older than 65 years, 64 patients were unable to complete the GHQ-30 questionnaire because of their mental status (confusion) $(n=24)$, physical illness $(n=20)$, or foreign language $(n=20)$. Moreover, 16 patients refused to participate in the study. The rate and kind of the excluded subjects were similar in ED and MD.

The GHQ-30 was completed by 719 subjects: 556 were admitted to ED and 163 to MD. Table l shows the sociodemographic features of the sample.

The rate of GHQ-30 positives patients was similar in ED $(47.4 \%)$ and in $\operatorname{MD}(53.9 \%)\left(\chi^{2}=0.8 ; d f=1 ; p=0.77\right)$. GHQ30 positive patients, compared with GHQ-30 negative patients, showed similar sociodemographic features in $M D$, but not in ED. In ED, GHQ-30 positives and negatives showed significant differences in sex distribution (female: GHQ-30 positives $59.7 \%$ versus GHQ-30 negatives $46.4 \%)\left(\chi^{2}=9.9\right.$; $\mathrm{df}=1 ; \mathrm{p}=0.008$ ) and in marital status (separated/divorced

Table 1 Sociodemographic characteristics of patients admitted to emergency department (ED) ward or to medical wards

\begin{tabular}{|c|c|c|c|c|c|c|}
\hline & \multirow{2}{*}{\multicolumn{2}{|c|}{$\frac{\text { ED ward }}{(n=556)}$}} & \multirow{2}{*}{\multicolumn{2}{|c|}{$\begin{array}{l}\text { Medical wards } \\
(n=163)\end{array}$}} & \multirow{2}{*}{\multicolumn{2}{|c|}{$\frac{\text { Total }}{(n=719)}$}} \\
\hline & & & & & & \\
\hline & Number & $\%$ & Number & $\%$ & Number & $\%$ \\
\hline \multicolumn{7}{|l|}{ Sex } \\
\hline Female & 297 & 53.4 & 78 & 47.9 & 375 & 52.2 \\
\hline Male & 259 & 46.6 & 85 & 52.1 & 344 & 47.8 \\
\hline \multicolumn{7}{|l|}{ Age } \\
\hline Mean (SD) & 39.7 (13.7) & & $49.1(12.8)$ & & $41.8(14.1)$ & \\
\hline \multicolumn{7}{|l|}{ Marital status } \\
\hline Never married & 198 & 35.6 & 28 & 17.7 & 226 & 31.7 \\
\hline Married & 313 & 56.3 & 104 & 65.8 & 417 & 58.4 \\
\hline Separated/divorced & 33 & 5.9 & 15 & 9.5 & 48 & 6.7 \\
\hline Widowed & 12 & 2.2 & 11 & 7.0 & 23 & 3.2 \\
\hline \multicolumn{7}{|l|}{ Family status } \\
\hline Living alone & 55 & 9.9 & 26 & 16.9 & 81 & 11.4 \\
\hline Living with someone & 501 & 90.1 & 128 & 83.1 & 629 & 88.6 \\
\hline \multicolumn{7}{|l|}{ Education } \\
\hline Primary school & 107 & 19.2 & 48 & 30.2 & 155 & 21.7 \\
\hline Secondary school & 168 & 30.2 & 65 & 40.9 & 233 & 32.6 \\
\hline College graduated & 240 & 43.2 & 35 & 22.0 & 275 & 38.5 \\
\hline University graduated & 41 & 7.1 & 11 & 6.9 & 52 & 7.3 \\
\hline \multicolumn{7}{|l|}{ Occupation } \\
\hline Unemployed & 14 & 2.5 & 4 & 2.5 & 18 & 2.5 \\
\hline Student & 40 & 7.2 & 7 & 4.4 & 47 & 6.6 \\
\hline Housewife & 64 & 11.5 & 23 & 14.1 & 87 & 12.2 \\
\hline Employed & 383 & 68.9 & 82 & 50.3 & 465 & 65.0 \\
\hline Retired & 55 & 9.9 & 43 & 27.0 & 98 & 13.7 \\
\hline
\end{tabular}


Table 2 Mental disorders in GHQ-30 positive patients admitted to emergency department (ED) ward or to medical department (MD) wards

\begin{tabular}{|c|c|c|c|c|}
\hline & \multicolumn{2}{|l|}{ ED } & \multicolumn{2}{|l|}{ MD } \\
\hline & Number & $\%$ & Number & $\%$ \\
\hline & 556 & 100 & 163 & 100 \\
\hline GHQ-30 positives & 264 & 47.4 & 88 & 53.9 \\
\hline Mental Disorders & 233 & 41.9 & 77 & 47.2 \\
\hline Anxiety Disorders & 101 & 18.1 & 19 & 11.6 \\
\hline Generalised Anxiety Disorder & 46 & 8.2 & 17 & 10.4 \\
\hline Panic Disorder & 31 & 5.6 & 2 & 1.2 \\
\hline Mixed Anxiety-Depressive Disorder & 15 & 2.7 & - & - \\
\hline Other Anxiety Disorders & 9 & 1.6 & - & - \\
\hline Mood Disorders & 64 & 11.5 & 34 & 20.8 \\
\hline Major Depression & 32 & 5.7 & 18 & 11.0 \\
\hline Dysthymia & 19 & 3.4 & 10 & 6.1 \\
\hline Minor Depressive Disorder & 7 & 1.2 & 3 & 1.8 \\
\hline Other Mood Disorders & 6 & 1.1 & 3 & 1.8 \\
\hline Adjustment Disorders & 49 & 8.8 & 18 & 11.0 \\
\hline Other Disorders & 19 & 3.4 & 6 & 3.6 \\
\hline
\end{tabular}

ED ward compared with medical wards (mental disorders were grouped in five categories: no disorders, depressive, anxiety, adjustment and other disorders): $\chi^{2}=11.7 ; \mathrm{df}=4 ; \mathrm{p}=0.02$.

or widowed: GHQ-30 positives $11.4 \%$ versus GHQ-30 negatives $4.6 \%)\left(\chi^{2}=10.7 ; \mathrm{df}=3 ; \mathrm{p}=0.01\right)$.

\section{Psychopathological evaluations}

The MINI was administered to 352 GHQ-30 positive patients (264 ED patients and $88 \mathrm{MD}$ patients). The rate of mental disorders was similar in ED patients $(\mathrm{n}=233 ; 41.9 \%)$ and in MD patients $(\mathrm{n}=77 ; 47.2 \%)\left(\chi^{2}=0.07 ; \mathrm{df}=1 ; \mathrm{p}=0.78\right)$.

ED and MD patients showed a significant difference in the rate of Anxiety Disorders (ED 20.3\% versus MD 14.1\%) and Depressive Disorders (ED $11.5 \%$ versus $20.8 \%) \quad\left(\chi^{2}=11.7\right.$; $\mathrm{df}=4 ; \mathrm{p}=0.02$ ) (table 2 ). No sex difference in the distribution of mental disorders was observed in $\operatorname{MD}\left(\chi^{2}=1.7\right.$; $\mathrm{df}=4 ; \mathrm{p}=0.78)$, whereas in ED female patients, compared with male patients, were more affected by depressive disorders $(25.6 \%$ versus $8.6 \%)$ and anxiety disorders $(35.2 \%$ versus $25.7 \%)\left(\chi^{2}=27.5 ; \mathrm{df}=4 ; \mathrm{p}<0.001\right)$.

At discharge, physicians diagnosed a mental disorder as main diagnosis in 21 of all the ED patients $(3.9 \%)$ and in seven of all the MD patients (4.2\%) (table 3). Moreover, a mental disorder was secondarily diagnosed in four ED patients $(0.7 \%)$ and in three MD patients $(1.8 \%)$. In all of these patients the presence of a mental disorder was confirmed by the MINI.

\section{Medical illnesses}

Table 3 shows the diagnoses at discharge (ICD-9 diagnostic categories).

The most frequent diagnoses at discharge were "injuries and adverse effects" (26\%) and "signs, symptoms, and ill defined conditions" $(22 \%)$ in ED patients and "diseases of circulatory system" (30\%), "endocrine, nutritional, metabolic disease" (almost all of them were diabetic) (13\%), and "neoplasm" $(9 \%)$ in MD patients. This difference reached significance $\left(\chi^{2}=178.6 ; \mathrm{df}=14 ; \mathrm{p}<0.001\right)$ (table 3$)$.

The medical disorders were more severe in MD patients (41.1 (SD14.7) than in ED patients $(29.4(14.3))(t=8.8$; $\mathrm{df}=717 ; \mathrm{p}<0.001)$. In both $\mathrm{ED}$ and $\mathrm{MD}$, no significant difference in the severity of the medical illness was observed between patients with or without mental disorders and among the categories of mental disorders.

Table 3 Diagnoses at discharge (ICD-9 categories) in patients admitted to ED or to MD wards

\begin{tabular}{|c|c|c|c|c|}
\hline \multirow[b]{3}{*}{ ICD-9 diagnostic category } & \multicolumn{4}{|c|}{ Diagnoses at discharge } \\
\hline & \multicolumn{2}{|l|}{ ED } & \multicolumn{2}{|l|}{ MD } \\
\hline & Number & $\%$ & Number & $\%$ \\
\hline Injuries and adverse effects (800-999) & 146 & 26.3 & 2 & 1.2 \\
\hline Signs, symptoms, and ill defined conditions (780-796) & 127 & 22.8 & 14 & 8.5 \\
\hline Diseases of genitourinary system (580-629) & 61 & 11.0 & 6 & 3.6 \\
\hline Diseases of circulatory system (390-459) & 56 & 10.1 & 49 & 30.1 \\
\hline Diseases of digestive system (520-579) & 46 & 8.3 & 14 & 8.5 \\
\hline Infective diseases (001-139) & 26 & 4.7 & 6 & 3.6 \\
\hline Mental diseases (290-319) & 23 & 4.1 & 7 & 4.2 \\
\hline Diseases of musculoskeletal system (710-739) & 21 & 3.8 & 10 & 6.1 \\
\hline Disease of nervous system and sense organs (320-389) & 19 & 3.4 & 3 & 1.8 \\
\hline Diseases of respiratory system (460-519) & 16 & 2.9 & 7 & 4.2 \\
\hline Endocrine, nutritional, metabolic disease (240-279) & 1 & 0.2 & 22 & 13.5 \\
\hline Neoplasm (140-239) & 9 & 1.6 & 15 & 9.2 \\
\hline Diseases of skin and subcutaneous tissue (680-709) & 4 & 0.7 & 3 & 1.8 \\
\hline Congenital anomalies (740-759) & 1 & 0.2 & - & - \\
\hline Diseases of blood and blood forming organs (280-289) & - & - & 5 & 3.1 \\
\hline All diagnostic categories & 556 & 100.0 & 163 & 100 \\
\hline
\end{tabular}

ED ward versus medical wards, $\chi^{2}=178.6 ; \mathrm{df}=14 ; \mathrm{p}<0.001$. 


\section{DISCUSSION}

This study investigated whether anxiety and depressive disorders differently affected patients admitted to ED or MD ward of a general hospital.

As expected, we observed a similar overall psychiatry morbidity in ED and MD wards, because in Italy the ED ward is an acute medical unit.

Nevertheless, anxiety disorders were more frequently diagnosed in the ED patients, whereas depressive disorders were more frequently observed in MD patients. This finding may be explained by the sociodemographic and medical characteristics of the patients admitted to MD or to ED: a higher number of MD patients were older, more frequently separated-divorced or widowed, living alone, housewife, or retired than ED patients. Moreover, MD patients showed a more severe medical disorder and a higher prevalence of cardiovascular diseases and cancer. All these features identified a population with a greater risk for depressive disorders. ${ }^{20}$ 29-31

This study also confirms our previous finding ${ }^{32}$ of a higher point prevalence of anxiety disorders $(21 \%)$ than depressive disorders $(10 \%)$ in ED patients.

The difference in psychiatric morbidity between MD and ED was attributable not only to the higher rate of panic disorder and mixed anxiety-depressive disorder in ED than in $\mathrm{MD}$, but also to the higher rate of major depression and dysthymia observed in MD than in ED.

These data agree with previous studies, ${ }^{11-13}{ }^{16}$ reporting that patients affected by panic disorder frequently seek ED for consultation. Also patients with mixed anxious-depressive disorder are known to be quite common in outpatient medical settings. ${ }^{25}$ Essential features of these disorders is dysphoric mood, lasting at least one month, and accompanied by concentration or memory difficulties, sleep disturbance, fatigue, irritability, hopelessness, low self esteem, or feelings of worthlessness. ${ }^{25}$

It is probable that the admission to ED ward for a diagnostic check up and treatment of mild somatic symptoms is requested for many anxious patients by ED physicians.

Differently from other anxiety disorders, generalised anxiety disorder showed a similar diagnostic rate in ED and MD, confirming that this disorder can also be frequently observed in patients with severe medical conditions. ${ }^{29}$

Even though depressive disorders were diagnosed more frequently in $\mathrm{MD}$ than in $\mathrm{ED}$, severe depression is quite common in ED patients, because it represents the third more frequent mental disorder. This finding confirms that depressive disorders are associated with an increased use of ED. ${ }^{24}$

Our data show that $4 \%$ of the patients were discharged with a diagnosis of mental disorder in either ED or MD. The rate of psychiatric diagnoses was surprisingly low particularly in ED, even though there was a high prevalence $(23 \%)$ of patients discharged with a first diagnosis comprised in the "signs, symptoms, and ill defined conditions" category (also named "unexplained physical symptoms"), which are frequently attributed to a psychiatric disorder. ${ }^{17}{ }^{33} 34$

The discrepancy between the frequency of psychiatric disorders diagnosed by the ED and MD medical staff and that found with the administration of the MINI could be explained by various reasons: (1) medical attention is particularly focused on life threatening illnesses or on medical diseases; (2) GH physicians may undergo medicolegal consequences if they fail to recognise medical conditions that mimic psychiatric disorders; (3) GH physicians might be insufficiently trained in psychiatry; (4) the GH medical staff might not have enough time for listening to psychological problems because of the large patient turnover; (5) GH physicians might consider depressive or anxious symptoms in severe medically ill patients as a normal psychological reaction.

Our data would suggest that training in psychiatric differential diagnosis would be important for GH physicians, and a strong collaboration between psychiatrists and GH physicians may lead to early detection and treatment of psychiatric disorders. Moreover, the recognition of psychiatric patients in GH can also be increased by the use of screening questionnaires such as GHQ-30 and Hospital Anxiety and Depression Scale, a self administered questionnaire with 14 items developed to evaluate anxiety and depression in patients with medical illness. ${ }^{35}$

Our study is limited by the choice of criteria in the assessment of the mental disorders in patients with medical diseases. The presence of symptoms, which may result from medical illnesses, might have increased the rate of falsepositive cases of mental disorders, as suggested by Cavanaugh $^{36}$ and Bukberg et al. ${ }^{37}$ However, the standardised interviews with specified criteria offer some advantages and are best suited for the diagnosis of mental disorders in the medically ill patients. ${ }^{20}$ Therefore, it is unlikely that the use of the MINI increased the prevalence of psychiatric diagnoses in our study.

Moreover, our data are not representative of a common medical inpatient population because our sample included only patients younger than 65 years and thus, the rate of mood disorders in older patients could not be evaluated.

Finally, our findings do not reflect psychiatric morbidity in the ED itself, because this study investigated mental disorders only in an ED ward.

In conclusion, our data confirm that anxiety and depressive disorders are frequently found in patients attending medical services. The ED, as one of the most promptly available medical facility, is more often frequented by anxious patients, even though severely depressed patients are also quite commonly discovered in this setting. The improvement in quality of care, the waste of healthcare resources through unnecessary medical care, and the fact that patients with anxiety and depressive disorders respond well to appropriate treatment (somatic treatment combined with psychotherapeutic support) make the diagnosis of these patients particularly important. Educational efforts will make effective treatment available to more patients.

\footnotetext{
Authors' affiliations

C Marchesi, E Brusamonti, C Maggini, Institute of Clinical Psychiatry, University of Parma, Parma, Italy

R Di Ruvo, F Minneo, Department of Emergency, Azienda Ospedaliera di Parma, Parma, Italy

A Giannini, C Quarantelli, Department of Medicine, Azienda

Ospedaliera di Parma

\section{REFERENCES}

1 Mayou R, Hawton K. Psychiatric disorders in the general hospital. Br J Psychiatry 1986;149:172-90.

2 Salkovskis PM, Storer D, Atha C, et al. Psychiatric morbidity in an accident and emergency department. Characteristics of patients at presentation and one month follow-up. Br J Psychiatry 1990;156:483-7.

3 Yingling KW, Wulsin LR, Arnold LM, et al. Estimated prevalence of panic disorders and depression among consecutive patients seen in an emergency department with acute chest pain. J Gen Intern Med 1993;8:231-5.

4 Ang AW, Ko SM, Kua EH. Psychiatric referrals from an accident and emergency department in Singapore. J Accid Emerg Med 1995;12:119-22.

5 Dula DJ, DeNaples L. Emergency department presentation with conversion disorder. Acad Emerg Med 1995;2:120-3.

6 Cherpitel CJ, Soghikinian K, Hurley LB. Alcohol-related health services use and identification of patients in the emergency department. Ann Emerg Med 1996;28:418-23.

7 Cavanaugh VS. The prevalence of emotional and cognitive dysfunction in a general medical population using the MMSE,GHQ and BDI. Gen Hosp Psychiatry 1983;5:15-24.
} 
8 Moore RD, Bone ID, Geller G et al. Prevalence, detection and treatment of alcoholism in hospitalized patients. JAMA 1989;261:403-7.

9 Cohen-Cole SA, Kaufman K. Major depression in physical illness: diagnosis, prevalence and antidepressant treatment (a ten year review: 1982-1992). Depression 1993;1:181-204.

10 Ballanger JC. Panic disorder in the medical setting. J Clin Psychiatry 1997;58(suppl 2):13-17.

11 Katerndahl DA, Realini JP. Where do panic sufferers seek care? J Fam Pract 1995:40:237-43.

12 Klein E, Linn S, Colin V, et al. Anxiety disorders in a general emergency service in Israel. Psychiatr Serv 1995;46:488-92.

13 Fleet RP, Dupuis G, Marchand A, et al. Panic disorder in emergency department chest pain patients: prevalence, comorbidity, suicidal ideation, and physician recognition. Am J Med 1996;101:371-80.

14 Barsky AJ, Delamater BA, Clancy SA, et al. Somatized psychiatric disorder presenting as palpitations. Arch Intern Med 1996;156:1102-8.

15 Hirschfeld RMA. Panic disorder: diagnosis, epidemiology and clinical course. J Clin Psychiatry 1996;57(suppl 10):3-8.

16 Tueth MJ. Managing recurrent nonischemic chest pain in the emergency department. Am J Emerg Med 1997;15:170-2.

17 Katon W. Panic Disorder: relationship to high medical utilization, unexplained physical symptoms, and medical costs. J Clin Psychiatry 1996;57/suppl 10):11-18.

18 Mayou R, Hawton K, Feldman E. What happens to medical patients with psychiatric disorder. J Psychosom Res 1988;32:541-9.

19 Mayou R, Hawton K, Feldman E, et al. Psychiatric problems among medical admissions. Int J Psychiatr Med 1991;21:71-84

20 Rodin G, Voshart K. Depression in the medically ill: an overview. Am J Psychiatry 1986;143:696-705.

21 Feldman E, Mayou R, Hawton K, et al. Psychiatric disorder in medical inpatients. Q J Med 1987;63:405-12.

22 Van Hemert AM, Hengeveld MW, Bolk JH, et al. Psychiatric disorders in relation to medical illness among patients of a general medical out-patient clinic. Psychol Med 1993;23:167-73.

23 Goldberg D. The detection of psychiatric illness by questionnaire. Maudsley monograph. London: Oxford University Press, 1972:21.
24 Marchesi C, Brusamonti E, Giannini A, et al. The use of an emergency ward by patients with depressive or anxiety disorders: a one year follow-up study. Int J Psychiat Med $2001 ; 31: 265-75$.

25 American Psychiatric Association. Diagnostic and statistical manual for mental disorders. 4th edn (DSM-IV). Washington DC: APA Press, 1994.

26 Bonora LI, Conti L, Piccinelli M, et al. Mini International Neuropsychiatric Interview (MINI) 4th edn. Italian version. In: Conti L, ed. Compendio di Psichiatria e Igiene Mentale. Florence: SEE, 1995:25-48.

27 Sheehan DV, Lecrubier $Y$, Janava J, et al. Mini International Neuropsychiatric Interview (MINI) release 4.4. Tampa, FL: University South Florida Institute of Research in Psychiatry and Paris: Inserm U302, Hopital de la Salpétrière, 1994.

28 Parkerson GR, Broadhead WE, Chiu-Kit JT. The Duke Severity of Illness (DUSOI) for measurement of severity and comorbidity. J Clin Epidemiol 1993:46:379-93.

29 Wells KB, Golding JM, Burnam MA. Psychiatric disorders in a sample of a general population with and without chronic medical conditions. Am J Psychiatry 1988; 145:976-81.

30 Johnson J, Wiessman MM, Klerman GL. Service utilization and social morbidity associated with depressive symptoms in the community. JAMA 1992;267: 1478-83.

31 Klerman GL. Depressive disorders. Further evidence for increased medical morbidity and impairment of social functioning. Arch Gen Psychiatry 1989;46:856-8

32 Marchesi C, Brusamonti E, Giannini A, et al. Psychiatric disorders in patients admitted to emergency department ward. Stress Health 2001;17:47-53.

33 Lloyd GG. Psychiatric syndromes with a somatic presentation. J Psychosom Res 1986;30:113-20.

34 Roy-Byrne PP. Generalized anxiety and mixed anxiety-depression: association with disability and health care utilization. J Clin Psychiatry 1996;57(suppl 7):86-91.

35 Zigmond AS, Snaith RP. The hospital anxiety and depression scale. Acta Psychiatr Scand 1983;67:361-70.

36 Cavanaugh S. Diagnosing depression in the hospitalized patient with chronic medical illness. J Clin Psychiatry 1984;45:13-16.

37 Bukberg J, Penman D, Holland JC. Depression in hospitalized cancer patients. Psychosom Med 1984;46:199-212. 